\title{
Meta
}

Journal des traducteurs

Translators' Journal

MONJEAN-DECAUDIN, Sylvie, dir. (2016) : La traductologie et bien au-delà. Mélanges offerts à Claude Bocquet. Arras : Artois Presses Université, 421 p.

\section{Maryvonne Boisseau}

Volume 62, numéro 3, décembre 2017

URI : https://id.erudit.org/iderudit/1043962ar

DOI : https://doi.org/10.7202/1043962ar

Aller au sommaire du numéro

Éditeur(s)

Les Presses de l’Université de Montréal

ISSN

0026-0452 (imprimé)

1492-1421 (numérique)

Découvrir la revue

Citer ce compte rendu

Boisseau, M. (2017). Compte rendu de [MonjEAn-DECAUdin, Sylvie, dir. (2016) : La traductologie et bien au-delà. Mélanges offerts à Claude Bocquet. Arras : Artois Presses Université, 421 p.] Meta, 62(3), 662-664.

https://doi.org/10.7202/1043962ar

Ce document est protégé par la loi sur le droit d'auteur. L'utilisation des services d'Érudit (y compris la reproduction) est assujettie à sa politique d'utilisation que vous pouvez consulter en ligne.

https://apropos.erudit.org/fr/usagers/politique-dutilisation/ 
interest not only to those TS scholars working on translation and ideology but to those in politicsrelated quarters, where, as Schäffner argues, TS should be taken rather more seriously.

María Calzada Pérez Universitat Jaume I, Castellón, Spain

Monjean-Decaudin, Sylvie, dir. (2016) : La traductologie et bien au-delà. Mélanges offerts à Claude Bocquet. Arras: Artois Presses Université, $421 \mathrm{p}$.

Comme son titre l'indique, cet ouvrage rassemble des articles écrits en hommage à Claude Bocquet, historien, épistémologue, psychanalyste et traductologue, professeur à l'Université de Genève de 1980 à $2011^{1}$. C'est, selon la présentation de Sylvie Monjean-Decaudin, un liber amicorum et c'est bien ainsi qu'il faut le lire.

Outre la préface, la liste des principales publications de Claude Bocquet et un entretien inédit, le volume compte dix-neuf articles traitant de sujets allant de la traduction à la traductologie, en passant par le discours scientifique, l'information juridique, l'histoire de la traduction et l'histoire. L'empan est large et l'on peinerait à y trouver un fil directeur si ce n'est dans l'hommage que chacun des articles rend à celui qui fut un pionnier de ce qu'on appelle aujourd'hui juritraductologie, une sous-discipline de la traductologie qui, compte tenu à la fois de sa spécificité, de son caractère interdisciplinaire et de son importance politique et sociétale, semble concentrer les problèmes auxquels tout traducteur et tout traductologue se heurtent, dans la pratique de la traduction et dans celle, secondaire, de la réflexion sur l'activité de traduction. Les articles ont été répartis en quatre sections d'environ une centaine de pages chacune. La première et la dernière traitent de questions à la périphérie du champ de recherche de Bocquet tandis que les deuxième et troisième parties, au centre de l'ouvrage, s'approchent véritablement du cœur de métier de Bocquet.

Intitulée De la traduction à la traductologie, la première partie rassemble cinq articles traitant de problèmes qui se posent, majoritairement, dans le champ de la traduction littéraire, que ce soit le rapport entre l'écriture fictionnelle et la traduction comme écriture, certaines stratégies de traduction nécessitant la mise en œuvre de procédés divers afin de préserver le contenu informationnel d'un texte ou le style. Ainsi, Corinne Wecksteen-Quinio ("Le traducteur: un écrivain refoulé») examine-telle la question du statut du traducteur suggérant l'hypothèse du traducteur comme écrivain refoulé. Elle s'appuie pour ce faire sur l'écriture de deux romans par deux traducteurs qui, chacun à leur façon, mettent en abyme la figure du traducteur en jouant sur le reflet inversé et déformé de l'image du traducteur dans le miroir de la fiction. L'interrogation sur l'image du traducteur est en fait présente dans tous les articles, de manière plus ou moins explicite. Catherine Delesse («Appellatifs et formes d'adresse dans Harry Potter et dans sa traduction française»), étudiant la traduction des termes d'adresse et la traduction du pronom you par «tu» ou «vous» dans la série des Harry Potter, met en lumière la plus ou moins grande adéquation des choix de traduction dans les situations pragmatiques d'interlocution tandis que Tatiana Alexeytseva («Explicitation par l'auteur et explicitation par le traducteur: quels parallèles?»), inspirée par Gérard Genette, montre que le traducteur n'échappe pas au travers de l'explicitation, terme qui englobe tout ce que le traducteur ou l'auteur ajoute à la périphérie du texte (préfaces, notes de bas de page, commentaires, etc.). Dans ce cas, la traduction offre un surplus informationnel facilitant la lecture alors que dans l'article de Desmond Gallagher ( Le contenu informationnel du texte d'arrivée »), ce sont toutes les entraves linguistiques et extralinguistiques à la restitution exacte du contenu informationnel qui sont recensées. Ces quatre articles renforcent, pour le lecteur, l'idée que la traduction est toujours imparfaite, ce que confirme le dernier article de cette première partie dont le sujet, la traduction du style, demeure une question insoluble. Cindy Lefebvre-Scodeller ( $« \mathrm{La}$ question du style en traduction littéraire») rappelle l'ancienneté de la question en balayant l'histoire de la traduction depuis l'Antiquité jusqu'à nos jours et plaide, en accord avec certaines tendances de la traductologie d'aujourd'hui, pour une vision plutôt «sourcière» de la traduction. De ces contributions prises ensemble se dégagent l'idée d'imperfection inhérente à la traduction et une certaine vision du traducteur, sensible à l'opinion que le lecteur peut avoir de lui. On regrettera, de façon générale, une structuration souvent déséquilibrée des articles eux-mêmes et, en particulier, dans l'article sur l'explicitation, l'absence de distinction conceptuelle entre explicitation et des termes comme explication ou commentaire. Enfin, dans le dernier article, on note une tendance à citer des auteurs à partir de sources secondaires quand le contexte même des citations originales aurait pu éclairer le propos d'une autre manière.

L'entretien avec Bocquet, qui ouvre la seconde partie (Du droit à la traduction juridique), constitue un des moments forts de l'ouvrage. En effet, le parcours même de Bocquet est à bien des égards exemplaire pour les chercheurs, qu'ils soient traductologues ou non: une formation initiale éclectique, une carrière à l'université après une 
expérience professionnelle de la traduction de textes juridiques dans une situation de trilinguisme, à l'origine de sa recherche guidée par des constats simples: "Toute traduction consiste à fixer un sens», dit-il p. 152, et une conscience aigüe de la marge étroite dans laquelle se meut la traductologie juridique, entre linguistique et traduction spécialisée ou littéraire, et dont l'apport peine à être reconnu par les juristes eux-mêmes. Les quatre articles qui suivent (Pierre Garrone, «La commission de Venise, pilier du patrimoine électoral européen »; Beat Bürgenmeier, «La mise en œuvre du concept de développement durable»; Christina Dechamps, "Traduction juridique et étude des collocations: quelles perspectives?»; Joaquin Giraldez Ceballos-Escallera, «Bases méthodologiques de constitution et d'exploitation d'un corpus juridique») abordent des problèmes plus techniques à travers la description du rôle d'une institution juridique et des études de cas. Ces travaux mettent en évidence la difficulté cruciale de l'interprétation en matière juridique et montrent la nécessité de recourir à des analyses interdisciplinaires prenant appui sur des connaissances juridiques et linguistiques des domaines considérés très approfondies et très fines, allant bien au-delà des seuls ouvrages lexicographiques et terminographiques. Dans tous les cas, «L'interaction entre les dimensions économique, écologique et sociale implique une connaissance approfondie de leur cadre institutionnel, qui est clairement défini par le droit international qui, à son tour, se trouve en interaction permanente avec les législations nationales qui s'en inspirent» (p. 196).

La troisième section de l'ouvrage (De la traductologie à l'épistémologie, quatre contributions), fort logiquement, élargit la perspective vers une des grandes questions de la traductologie, à savoir l'éthique de la traduction, l'éthique du traducteur, la déontologie du métier. Teresa Tomaszkiewicz («Le rôle des codes d'éthique professionnelle dans la formation et dans l'exercice du métier de traducteur. L'exemple polonais»), après avoir constaté l'absence de formation à une éthique professionnelle dans les formations de traducteurs existantes en Pologne et dans d'autres pays d'Europe, réfléchit sur les rôles multiples des traducteurs et interprètes particulièrement exposés dans des situations conflictuelles. Elle préconise de passer d'une éthique en général à une éthique en situation: «... une éthique du traducteur doit consister tout d'abord à bien pondérer entre ce qui est personnel et ce qui est social, entre ce qui est utile et ce qui nuit à l'interaction, entre ce qui facilite et ce qui bloque l'intercompréhension» (p. 252). L'analyse par Catherine Anaïs Bocquet, dans l'article suivant ( «La non-traduction entre courage et compétence professionnelle»), de situations dans lesquelles la «non-traduction» - qui revêt des formes diverses - peut être assimilée à un acte de courage renforce l'idée d'une formation à l'éthique, partie intégrante de la compétence du traducteur. La problématique développée dans le troisième article de cette section ne présente qu'un lien ténu avec celle de la traduction. Son intérêt réside dans la remise en cause de l'objectivité et de la neutralité de la langue scientifique (à partir de l'exemple des sciences expérimentales) tout autant que dans la mise en lumière du danger d'une langue métaphorique, la métaphore conceptuelle étant conçue comme un modèle (Mikhail Chakhparonian, «Sciences expérimentales: quelques réflexions sur leur langage et sur leur enseignement»). Jean-René Ladmiral clôt cette troisième partie de l'ouvrage avec un article interrogeant à la fois l'idée de «fondement» et celle d' "épistémologie» de la traductologie. Ladmiral défend l'idée d'une épistémologie coextensive au développement de la discipline, cette discipline de «second rang» (p. 307). Selon lui, le discours traductologique est foncièrement un discours subjectif et ce qu'il appelle "méta-théorie» doit être en prise directe sur la "pratique traduisante»: «Pas plus que de fondements épistémologiques, il n'y a de règles a priori: car dans une pratique, tous les problèmes se posent en même temps» (p. 313). Ce qui relie ces quatre contributions tient sans doute à une forme de critique de l'objectivisme de la réflexion traductologique appliquée à «l'acte traductif» et à «l'activité traduisante».

Les six contributions de la dernière partie de l'ouvrage, de longueurs diverses, relève de l'histoire (égyptologie: Pierre Meyrat, "Copie conforme. Traduction et diplomatie dans l'Égypte ancienne»), de l'histoire de la traduction (Claudia Wick, «Jérôme et la bible latine: révision critique ou (re) traduction?»; Marcel Picquier, «Les vraies raisons de la condamnation à mort d'Étienne Dolet»), à laquelle on rattachera un questionnement sur la censure ou l'autocensure (dont Ballard dit qu'elle pourrait bien être une qualité du traducteur) que des retraductions peuvent révéler (Michel Ballard, «Joseph travesti: un coming out difficile!»). Ces articles rappellent tout à la fois l'ancienneté de la traduction, le rôle éminemment politique de la traduction et de l'interprétation, les querelles susceptibles de naître de positions non conformes aux idéologies dominantes quelle qu'en soit leur nature, et les risques encourus par les défenseurs de la liberté d'expression. Les deux derniers articles (Luc Weibel, «Un étudiant genevois à Iéna; Ruth Fivaz-Silbermann, "Grand résistant et juste méconnu: le docteur Paul Bonier de Saint-Julien, 1911-2003) racontent deux parcours individuels très dissemblables: celui de Charles Borgeaud, étudiant genevois ayant fait un doctorat à Iéna avant de revenir enseigner le droit et l'histoire à Genève 
et celui du docteur Paul Bonier dont la famille était connue de celle de Claude Bocquet, et qui, dans les années 1940, a aidé et sauvé des Juifs en faisant en sorte qu'ils puissent passer en Suisse depuis l'hôpital où il exerçait, à Saint-Julien-en-Genevois. La présence de ces articles, apparemment «hors sujet» (p. 409), pour reprendre le qualificatif utilisé par Fivaz-Silbermann elle-même, est toutefois éminemment pertinente dans cet ensemble où la mise en lumière de l'importance des frontières et des passages, du rôle des interprètes et des traducteurs, passeurs eux-mêmes, vient renforcer l'idée que l'activité de traduction ne se fonde que sur des valeurs humanistes d'honnêteté, de rigueur, de respect du texte à traduire, et de considération envers le lecteur auquel est destiné le texte traduit, et cela en dépit d'une censure rampante faite notamment de contraintes éditoriales, de normes sociales ou d'interdits que l'on se donne à soi-même.

La variété des approches et des thèmes abordés, le respect, l'affection et l'amitié envers celui qui fut un pionnier de la juritraductologie dont chacun des articles témoigne, justifient pleinement le titre de l'ouvrage, La traductologie et bien au-delà. Il $y$ est en effet question d'une pratique professionnelle dans toutes ses dimensions, intellectuelles, pragmatiques, éthiques et, sans aucun doute, le lecteur, spécialiste ou non, non seulement enrichira ses connaissances mais y trouvera ample matière à réflexion.

Maryvonne BoIsseau Université de Strasbourg, Strasbourg, France

\section{NOTE}

1. Un colloque international avait, préalablement à cette publication, rassemblé les 5 et 6 mai 2011 les auteurs de ces articles à l'Université de Genève. Deux articles ont d'ailleurs fait l'objet d'une première publication à la suite de ce colloque dans la revue Parallèles, n ${ }^{\circ} 25,2013$ (revue de la Faculté de traduction et d'interprétation de l'Université de Genève).

TAKedA, Kayoko and BAigorri-JALón, Jesús, eds. (2016): New Insights in the History of Interpreting. Amsterdam/Philadelphia: John Benjamins, $278 \mathrm{p}$.

Due to the evanescent nature of interpreting and generally subordinate status of interpreters as agents in historical events, interpreting activities have seen much fewer records than translation and even less research on their history. This new book entitled New Insights in the History of Interpreting is a valuable collective effort in locating the few references to interpreting and interpreters in historical archives and personal accounts and in analyzing how interpreting has been practised over time and space as well as issues involved in it.

The book is a themed volume of ten original articles about interpreting and interpreters in inter-lingual and cross-cultural communication through history. Collecting eight papers from the international symposium on interpreting history that was held at Rikkyo University in 2014 and two other select pieces, this volume records and discusses interpreting practices, recruitment of interpreters, and issues and challenges that interpreters have faced in diplomacy, colonization, religion, war, and occupation in different historical periods and in various regions of the world such as East Asia, America, the U.S.A. and the Soviet Union.

One major value of historical study is to discover 'new' historical facts hidden in the vast number of archives and to make new interpretations of "old" historical facts. This book represents a good effort in providing new insights in the history of interpreting across different regions of the world.

Four chapters (Chapter 1, 4, 8 and 9) provide new discoveries about interpreting history in East Asia. Rachel Lung, who is well-known for her research on interpreting history in Early Imperial China (Lung 2011), defines the multiple roles of Sillan interpreters in first-millennium East Asian exchanges. In an attempt to clarify the idiosyncratic title of "Sillan (ancient Korean) interpreters," she identified the thirty-eight references to Sillan interpreters in Japanese monk Ennin's (794-864) travelogue of his China sojourn (838-847). Her chapter outlines finer categories of these interpreters, whose duties were multifarious: including not only language mediation but also liaison; networking; coordination and marine travel consultancy. She argues that such multiplicity in tasks and roles points to a major distinction between official interpreters and civilian interpreters. Torikai Kumiko introduces Oranda Tsūji, a group of Dutch interpreters in Japanese history, as depicted in four historical novels written by Yoshimura Akira, which explore the life of Nagasaki Tsūji in the social context of pre-modern Japan during later years of the Edo period. As a step furthering her research on interpreting activities in the Tokyo War Crimes Tribunal (Takeda 2010), Takeda describes the diverse experiences of Japanese interpreters in the post-World War II occupation period (1945-1952): Some interpreted for the war crime trials; others were persecuted as war criminals; and others served foreign military occupiers. The research reveals issues and risks faced by wartime interpreters and local interpreters serving foreign military occupiers, which points to the vulnerable 